10 years ESJ

Special edition

\title{
Preface: Communicating, Educating, Interfacing during COVID-19 Pandemic
}

\author{
Franca Daniele, $M D$ \\ Department of Medical, Oral and Biotechnological Sciences \\ "G. d'Annunzio" University, Chieti-Pescara, Italy
}

Doi: 10.19044/esj.2021.v17n30p1

Published: 10 September 2021

Copyright 2021 Author(s)

Under Creative Commons BY-NC-ND

4.0 OPEN ACCESS

Cite As:

Daniele F. (2021). Preface: Communicating, Educating and Interfacing during COVID-19 Pandemics. European Scientific Journal, ESJ, 17 (30), 1.

https://doi.org/10.19044/esj.2021.v17n30p1

This Special Edition of the European Scientific Journal - ESJ results from the speeches that were held at the online Multidisciplinary Conference on May 17. The Conference had several Special Sessions and I had the honor to chair the session on Medical and Health communication, especially during a pandemic.

Health communication involves communicating health information to the general public during public health campaigns, all types of health education events, and between doctor and patient. The purpose of spreading health information is to affect and possibly improve health choices by the general public, due to better understanding of health issues (Schiavo, 2013). Health communication is a special field in healthcare that allows professionals to inform and impact individual and community decisions and actions, with the goal of improving and enriching personal and community behaviors and public health practices (Thomas, 2006). Like in all types of communication interactions, three elements are involved: the message, the target and the communicator. An effective health communication must be specifically adapted for the target and the situation, research into health communication seeks to refine communication strategies to inform people about ways to enhance health or to avoid specific health risks (Kreuter \& Wray, 2003). Health communication is also a scholarly discipline in the broader field of communication. Researchers in universities all over the world investigate how messages are created, shaped, and received within different contexts, such as age, ethnicity, gender, sexual or political orientation. Researchers in the field 
of communication may be concerned with how interpersonal communication affects people's attitudes and beliefs around medical issues (Rogers, 1992).

One of the main elements of effective interactions is the communicator, and in this context, Kirk St.Amant, our keynote speaker presented his research on mind processes and communication. He revealed that: "Cognition - or how the mind processes information - often guides human behavior in familiar settings. Such factors can affect the communication practices persons use to convey information about health concepts or medical processes. The challenge becomes anticipating such connections in order to create texts and visuals audiences can understand and use to meet healthcare needs. This article presents an approach to meeting this need by applying the concept of cognitive scripts to understand and address the communication expectations audiences often associated with health and medical settings. In presenting these ideas, the article begins with an explanation of how cognitive scripts can affect communication processes. From there, the author advocates applying script dynamics to health and medical communication practices. To do so, the author expands upon ideas in the literature on cognitive scripts to create a script-based approach for researching an audience's expectations of healthcare situations. The author then describes how to apply the information collected from this research to create communication materials audiences can more easily use in healthcare contexts. The result is a three-factor method that focuses on applying cognitive scripts to identify and address an audience's expectations for healthcare communication in a given context. Readers can then use this approach to design healthcare communication materials that audiences can easily and effectively use".

Second, any communicative message must be linguistically accurate, correct and clear, meaning that the content is free of mistakes, can be understood, interpreted and judged by an audience sharing the same language. In this specific regard, I presented an analysis on medical abstracts, which represent the source of all medical information: "Medical communication and health communication explaining that they are two close relatives in the field of communication, where medical communication is the mother and health communication is the offspring. Medical communication engages the delivery of scientific, medical, pharmaceutical and biotechnological information and data to health professionals like doctors, pharmacists, nurses, etc. The information includes updates on the latest discoveries provided by the international scientific community. Therefore, the source of this type of communication is represented by medical and scientific publications reporting data generated from basic science and clinical research. Health communications are targeted toward the general public, where the source is represented by health communicators and journalists. In health 
communications, information is the result of some kind of intra-language translation that allows transformation of the original medical language into a common language. Therefore, health communication derives from rewritings of a complex medical language that cannot always be modified and acquainted to serve the general public. The aim of the present work was to evaluate, in medical communications, the linguistic elements that represent the hard core for the general public. Thus, a qualitative evaluation was carried out on medical abstracts assessing medical terminology and compound phrases. The results of this investigation point out that these two linguistic traits of medical language are especially difficult for the general public due to their particular specialized nature".

Besides being linguistically correct and understandable by the target, the message should lack cultural, race, gender and religious implications and should be balanced, providing different and valid perspectives on the issue, as the source of the content is credible and updated (Kreuter \& McClure, 2003). The vehicle through which the message is carried is paramount, as it should be made available to the largest possible number of people in the target, meaning that it has been delivered where the target can access it, it should be repeated and consistent in time.

The second element playing a role in effective interactions is the target the message is addressed to. In this context, health literacy is crucial and can be defined as the capacity that an individual has to access and effectively use health-related information, in order to promote and maintain good health. Health literacy can enable people to understand and communicate health information and concerns. Examples of actions that require health literacy skills include properly reading and adhering to a care or prevention program, being able to use the available healthcare services rationally and ponder individual behavioral change, especially during exceptional conditions like a pandemic. Increasing health literacy rates is a means to empower people and contribute to downgrading inequalities towards a healthier, safer, more demanding society (Kickbusch et al., 2013). In these specific contexts, Barbara Cappuzzo carried out a survey on the websites of the major Italian healthcare institutions to find out how the COVID-19 pandemic issues were communicated to non-Italian speakers: "Health is a common issue for all human beings. As a consequence, everyone in the world has in some way to cope with the language of medicine. This is true now more than ever due to the global health crisis caused by the current COVID-19 pandemic, which has introduced a great amount of terms, previously mostly used by epidemiologists and statisticians, but which now have entered the daily lexicon of many languages. As the medium of international scientific communication, English is the language of worldwide information about the pandemic, and the main source of terms and expressions for other languages. 
The impact of the COVID-19 pandemic on English lexicon has been so deep that the Oxford English Dictionary Online released special updates in 2020 to fulfil the need to document the phenomenon. However, previous studies have highlighted the important question concerning the existence of several ethnic minorities who have Limited English Proficiency and therefore do not receive sufficient and appropriate information to defend themselves adequately against SARS-CoV-2, the virus we have all been fighting for more than one year now. The aim of this study is to highlight the importance of language and translation as essential components to provide all demographic groups/communities with access to COVID-19-related information in languages other than English and enable them to follow official health key rules. The main websites of Italian governmental and non-governmental institutions were investigated, and the analysis focused on the availability and type of content of the multilingual material, as well as on information accessibility and clarity. The results showed important differences in the number of available languages and, even more, in the level of intelligibility of COVID-19 material in the English language. In this respect, this study intends to foster the use of plain English in the dissemination material provided by the websites of the main healthcare public institutions in Italy, a country with an ever-increasing number of registered foreigners, the majority born in non-EU countries".

Health communication is a field that is developing apace, includes not only patient/doctor communication and health education, but also health and policy advocacy. Advocacy is a strategy used to increase familiarity with issues regarding promotion of health and access to quality health care and public health. Health advocacy may also help guide health policy reforms, and it may be done utilizing different vehicles, such as: mass and multimedia, direct political lobbying and community mobilization (Hubinette et al., 2017).

One of the principal goals of health education is to inform people about lifestyles and behaviors that help prevent diseases. In this sense, health education aims to positively affect people's awareness, attitudes and behaviors related to their health. It is a process during which people learn how to take care of theirs and other people's health. Health communication includes doctor/patient interactions, as well as community-focused communications, such as public meetings, local newspaper advertisements, pamphlets, or educational events (Daniele, 2007). It also includes mass media communication, through social media, internet, television, and radio messaging to reach the general public with information about preventative health care, disease prevention and treatment, immunizations, and more (Haux, 2006). Health communication plays a significant role at all levels of disease prevention and health promotion, increasing knowledge and awareness of a health issue and to affect beliefs and attitudes, as well as 
showing the benefits of behavior change. Meanwhile, patients benefit from increased access to their medical histories, which reduces chances of medical errors. Health communication strategies allow doctors and nurses, community health centers, hospitals and hospital administrators, nursing homes, health educators, nonprofits, government agencies, and others to disseminate information that can positively influence personal health behaviors and choices (Daniele, 2007). Audrey Tolouian, Diane Monsivais and Melissa Wholeben spoke about being nurses and nurse educators during this COVID19 pandemic: "Nurse educators who are novice often express the need for improved preparation in the educator role. With the growth of online programs, one area of concern is the need for educators who are prepared to teach online. A mentorship for online teaching was developed to give student nurse educators the opportunity to develop educator competencies in the online environment. The process, benefits, challenges, and key points for the success of the mentorship are discussed. Since Spring 2016, 89 nurse educator graduate students have completed the mentorship. Their confidence related to the educator role in an online environment was enhanced, and they took great pride in serving as professional role models to the undergraduate nursing students. The mentorship option provides improved educator role preparation for nursing education graduate students who will teach online. It also provides informal mentorship as well as an unexpected perceived value in higher education to students in undergraduate nursing programs".

During the outbreak of COVID-19, companies and the entire productive system all over the world had to take specific measures to acquaint themselves with the new situation. Daniele Di Lorenzo reports on changes in project management during a crisis: "A crisis induces project managers to use, increasingly, a specific "language" that is born from the union of two dimensions, technical-operative modalities and the mindset. The goal is to properly react to the crisis, like a pandemic scenario will. This approach directly involves the usage of "triad" (Reaction-Execution-Results) and micro-goals concepts in order to maximize the effectiveness and efficiency of the project manager actions within time windows. Project management is one of the most important disciplines in a company, especially where the project is the soul of the business and the center of the employees' dynamic. This paper focuses on the "interfaces" between project management and other company functions. It analyzes how the "language" could induce reaction behavior based on the time-constraints and micro-goal modus operandi and how the company dynamic could change its behavioral habits towards the same common company purpose: the success of projects".

The Conference included another extremely interesting session on Law in Georgia, and Ekaterine Nandoshvili "focused on analyzing the norms regulating joint rights, presents their shortcomings, and criticizes the 
misconceptions expressed in the legal literature about the types of common property, joined rights, and co-ownership. The paper considers the incompleteness of the provisions regulating the legal consequences of the abolition of joint rights as a serious shortcoming of the Civil Code of Georgia. A novelty is a mechanism proposed in this paper, and it is possible to assign the entire property to one of the participants in case of abolition of joint right, in exchange for compensation for the shareholder who requests the abolition of joint right and the allocation of the amount. The objective of the paper is to analyze certain aspects of the regulation of joint rights, which, together with the theoretical, have the practical importance that will contribute to a correct understanding of a number of issues and the correct qualification of the rights and obligations of participants of the legally binding relationship, rising on the basis of joint rights. To achieve this objective, logical and systematic analysis of norms as well as comparative-legal methods are used. Using these methods, it is possible to determine the strengths and weaknesses of the norms of Georgian law or to better understand their content, to identify gaps in the legislation in court practice, as well as to develop proposals and recommendations for the improvement of norms and practice. The problems are analyzed using the examples of Georgian, German, and Swiss civil law. The common features and shortcomings were identified between the Georgian and German models regarding the issue of the consequences of the abolition of joint rights. The Swiss model appears to be the most perfect and effective model among the named ones. The study found that neither in practice nor under the law is a shareholder allowed to sell the joint item in an auction by redeeming the shares of other owners. The extinction of this opportunity for the owner reduces the essence of ownership. In order to extend the guarantees for full protection of the property rights, a view is proposed on the need to develop an approach, similar to the Swiss model, and the implementation should be ensured by case law before the law is changed".

The Conference and this Special Edition have been a valuable professional and human experience. I thank all of my Colleagues and the wonderful ESI team for having made it happen.

\section{References:}

1. Daniele F. (2007). "Bridges to Language Barriers". In: Cityscapes the Islands of the Self, Proceedings of the Associazione Italiana di Anglistica, Cagliari, pp. 173-184.

2. Haux R. (2006). Health information systems - past, present, future. International Journal of Medical Informatics, Volume 75, Issues 3-4, Pages 268-281.

https://doi.org/10.1016/j.ijmedinf.2005.08.002. 
3. Hubinette M., Dobson S., Scott I., Sherbino J. (2017). Health advocacy. Medical Teacher, Volume 39, Issue 2 Pages 128-135.

4. Kickbusch I., Pelikan J.M., Apfel, Franklin \& Tsouros A. (2013). Health Literacy. WHO Regional Office for Europe. https://apps.who.int/iris/handle/10665/128703.

5. Kreuter M.W. and McClure S.M. (2004). The Role of Culture in Health Communication. Annual Review of Public Health, Volume 35, Pages. 439-455.

6. Kreuter M.W. and Wray R.J. (2003). Tailored and Targeted Health Communication: Strategies for Enhancing Information Relevance. American Journal of Health Behavior, Volume 27, Supplement 3, November, pp. S227-S232(6).

7. Rogers E.M. (1994). The Field of Health Communication Today. American Behavioral Scientist. Volume 38, Issue 2, Pages 208-214. https://doi.org/10.1177/0002764294038002003.

8. Schiavo R. (2013). Health Communication: From Theory to Practice. Wiley.

9. Thomas R.K. (2006). Health Communication. Springer. 